\title{
Harmonic Mitigation Technique using Active Power Filter Controller for Textile Industry
}

\author{
Afthab Zacker Hussain, Jimmy Jain George, Vijay .N, William V Shelly
}

\begin{abstract}
One of the major power quality issue that industries face is harmonic distortion. Non-linear loads are the major source of harmonics. Textile industries incorporate a large number of non-linear loads which introduces harmonics to the system and finally leads to waveform distortion. Harmonic glitches in the industry are produced from non-linear loads such as rectifiers, variable speed drives etc. The harmonics distortion is a form of electrical pollution that can cause problems for both utility and customers if the sum of the harmonics current increases above certain limits. Harmonics may cause a lot of complications such as improper measurement of the electricity meters, failure of protective devices, ageing, transmission lines additional losses, nuisance tripping of circuit breakers and damaging of electric devices. In this paper, an active power filter scheme is developed as a solution for harmonic distortion. The developed APF control scheme is based on a proportional feedback controller and a single phase voltage source full wave bridge rectifier. By reducing harmonics, the system efficiency can be made optimum. It avoids malfunctioning of equipment's, reduces losses, heat, vibrations, maintains the power factor within the acceptable IEEE standard values and further reduces total harmonic distortion.
\end{abstract}

Index Terms - Active Power Filter, Harmonic Analysis, Textile Industry, Total Harmonic Distortion, UCC28180 Controller.

\section{INTRODUCTION}

In any industry, one of the major concerns is to minimize the electrical losses and ensure efficient operation of its electrical infrastructure. Just like any other industry, the textile industry has a very high usage of power converters owing to their high efficiency. In the modern textile industry solid state devices are extensively used for various applications such as rectifiers, automation system, flexible control of speed and power etc. Meanwhile, these solid state devices are non-linear components either element in which current is drawn in abrupt short pulses where the current is not proportional to voltage. Harmonics are the result of such non-linear loads. Thus the power quality issue of waveform distortion arises in the power system. The converter systems can generate both characteristic as well as non-characteristic harmonics such as interharmonics. The harmonics tolerance limits for various devices are defined by the IEC standards. In

Afthab Zacker Hussain, Rajagiri School of engineering and Technology, APJ Abdul Kalam technological University, Ernakulam, India

Jimmy Jain George, Rajagiri School of Engineering and Technology, APJ Abdul Kalam Technological University, Ernakulam, India

Vijay .N, Rajagiri School of Engineering and Technology, APJ Abdul Kalam Technological University, Ernakulam, India

William V Shell, Rajagiri School of Engineering and Technology, APJ Abdul Kalam Technological University, Ernakulam, India a textile plant nearly half of the total power is consumed by the variable frequency drives used for various applications such as yarn dyeing, fabric dyeing, blowers etc.

Here, an active power filter controller is designed to mitigate the issue of harmonic distortion in the textile industry power system. Many devices require an AC to DC conversion which is done with the help of a rectification unit. But the switching devices of the rectifier constitute non-linear load that introduces harmonics to the system. To solve this issue the output from the rectifier is fed to a boost converter whose switching is controlled by means of a power factor correction controller. The controller used here is UCC28180, 8-pin CCM PFC Controller, which is a dedicated IC for power factor correction. Power factor controller takes the pure sinusoid as one of the input and the output voltage is also fed to the controller. The system ensures that the sinusoidal nature of the waveform is preserved despite of the harmonic injection from the rectifier. A single phase supply is used here, as the system is designed to meet a low load but can be implemented for a three phase system as well.

\section{LITERATURE SURVEY}

This paper presents the harmonics and the different problems caused by the harmonics injection into the power systems in a textile industrial point of view. In this paper, we had mainly focused on the different harmonic problems in a textile industry and proposed a system which could help reduce the harmonics which are being injected in textile industries mainly due to the number of VFD's being used.

Periyaazhagar .D and Irrusapparajan .S, put forward a paper which discusses about the harmonic distortion and reduction techniques. The paper mainly discuss about the different types of loads which can contribute to the injection of harmonics into the system. The paper presents different non-linear loads, such as, variable speed drives, arcing devices, fluorescent lamps, personal computer, and the waveforms which are generated by these loads. The size as well as the location of non-linear loads plays an important role in the operation, maintenance as well as troubleshooting process. Various Methods were also discussed in the paper which could help level the different effects of harmonics. The different impacts and changes that the harmonics can impose onto a system were discussed. [1]

H. Mageed, A. S. Nada, S. Abu-Zaid, R. S. Salah Eldeen, presented a paper which highlights about the harmonic problems which were caused by different household appliances. They investigated the different impacts of harmonics which were generated mainly by the household appliances. They used a CALMET-TE30 three phase power 
quality analyzer for their experimental measures. The electronic household appliances produced high distortion in their voltage and current waveforms. It is clearly defined by the different waveforms that the power factor is greatly affected due to the presence of harmonics. They had also analyzed these effects on a seasonal level, as the waveforms show more distortion in the summer when compared to winter. The paper provides a recommendation of providing an active filter in parallel with the household appliances to mitigate the harmonic effects. [2]

Roohi Sachan and Rohan Srivastava presented a paper which mainly deals with the designing and implementation of fixed passive filters for the mitigation of harmonics. The performance of the filters was checked for varying firing angle provided to a thyristor controlled converter. These proposed filters work efficiently and satisfactorily when the reactive power demand is fixed. [3]

Henning Tischer and Tomaz Pfeifer provide us with a case study of hybrid filter which was installed in a metal processing plant. This filter is a combination of an active filter and a passive voltage notch filter which is realized through resistors and capacitors. It provides the system with dynamic harmonic filtering and helps in reducing voltage notches in a low voltage network. [4]

V. Suresh Kumar and V. Saravanan presented a helpful case study on harmonic distortion in a typical textile industry. The case study was conducted in a textile plant at Dindigul district of Tamil Nadu state in India. In a textile industry, energy consumption is going on increasing, due to the modernization of machines and continuous usage of different equipment's in inefficient operating ranges. Nowadays the area of focus is towards energy consumption at the load side and optimizing the efficiency of different motor drives. Usually the textile mills can be divided into three systems namely, card silver, combed silver and ring spinning system. So there is a need for a large number of machines in these different sections. Also the performance of the machines must be variable for the different applications in textile industries. For this, the motors are provided with variable frequency drives (VFD's) which helps in plant control operations. These VFD's are one of the major sources of harmonics in textile industries. This paper also analyses the waveforms produced by the VFD's used in varying processes at different loadings ranges. Let us look at the level of harmonics being injected into the system due to a $150 \mathrm{~kW}$ VFD used in fabric dyeing unit.

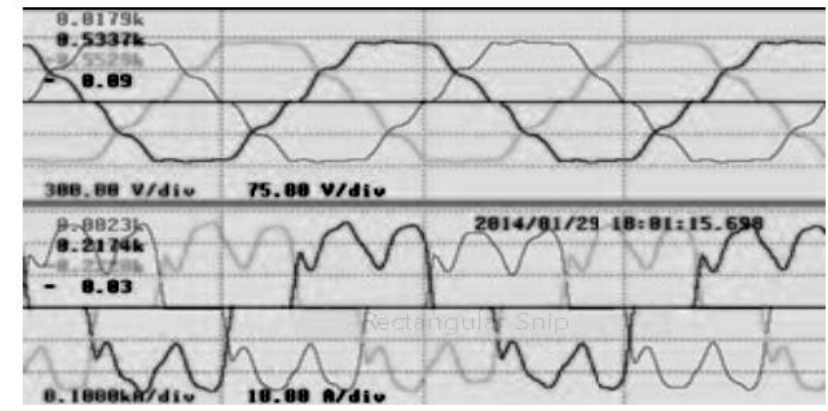

Fig.1. Voltage and Current waveform of $150 \mathrm{~kW}$ VFD for fabric dyeing unit [5]

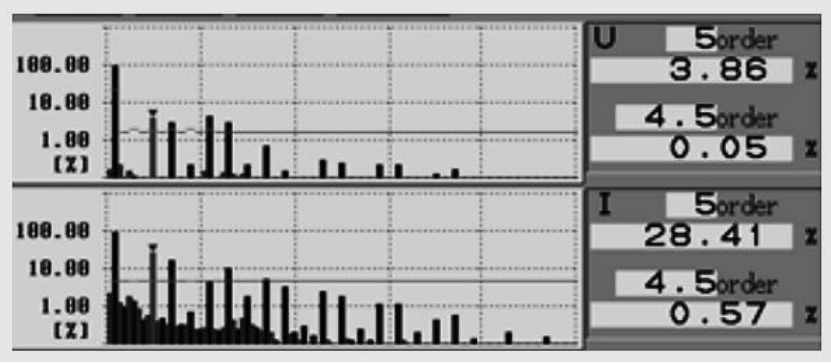

Fig.2. Harmonic Spectrum of 150kW VFD [5]

The voltage and current waveform of a $150 \mathrm{~kW}$ VFD used in the fabric dyeing unit is shown in Fig. 1 when it is loaded for $110 \mathrm{~kW}(73 \%)$. The VFD drive system consists of a rectifier and IGBT based PWM inverter. Fig.2 displays the harmonic spectrum for the analyzed waveform of $150 \mathrm{~kW}$ VFD. Observe that the $5^{\text {th }}$ harmonic component for the current waveform is $28.41 \%$ of the fundamental component. [5]

\section{PROBLEM STATEMENT}

As a textile industry covers a lot of non-linear load ranging from power converters, variable speed drives to uninterrupted power supplies and computers; it imparts a huge amount of harmonics to the supply. This can reduce the power factor, increase the losses and can affect the operation of sensitive equipment's. High amount of harmonics can badly affect the performance of distribution transformer in a textile industry. Hence, a cost effective and efficient solution has been brought which could minimize the effect of waveform distortion due to harmonics at the load end.

\section{SYSTEM ARCHITECTURE}

\section{A. System Block Diagram}

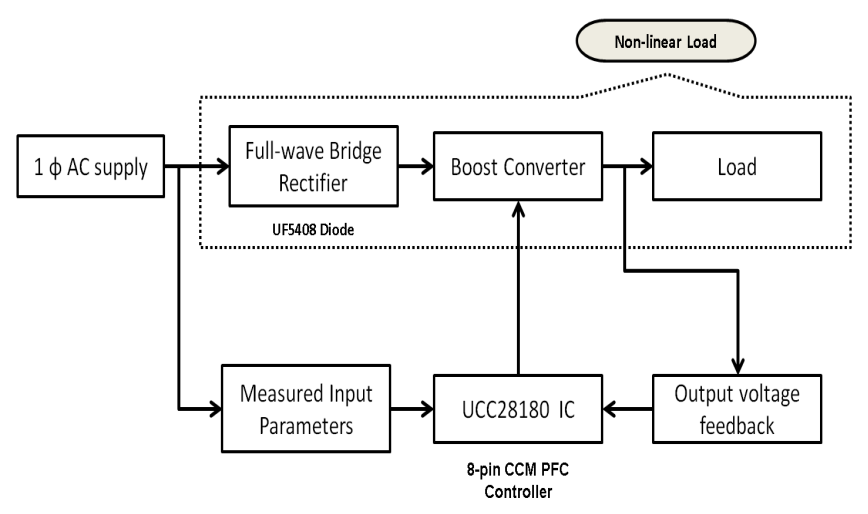

Fig.3. Block Diagram of the system

In our model block diagram, a single-phase ac supply at standard frequency is rectified with the help of full wave bridge rectifier. The rectified DC output is provided to a boost converter which is made to operate in continuous conduction mode (CCM). The boost converter is introduced to provide the filtering action for the harmonics. The voltage is boosted to $400 \mathrm{~V}$ to meet a load of $100 \mathrm{~W}$. The input voltage waveform is considered as the reference signal for the gate driving signals to be generated. The distortion produced by the rectifier part is controlled by means of UCC28180 power factor controller (PFC) IC which accordingly sends switching pulse to the gate of the boost converter switch. The output 
voltage is fed back to the controller to provide the voltage control feedback loop. The gate driving circuit of the controller sends pulses at high frequency to preserve the shape of the waveform. It does not allow the respective waveform to shift to a value above or below as compared to the reference wave. The 8-pin CCM PFC Controller takes two inputs, one is the reference waveform from the input side and the other is the output voltage. The controller has a wide range of switching frequency to support various switch devices.

\section{1) Rectifier Block}

The AC-input voltage is converted into uncontrolled DC voltage using diode rectifier block. To reduce the voltage stresses across the switches to $\mathrm{V}_{\mathrm{m}}$ a Diode bridge rectifier is used. The ripple in output of rectifier is reduced by connecting the capacitor across the output.

\section{2) Boost Converter Block}

Boost converter block consists of mainly the Boost inductor, MOSFET and diode. The MOSFET switching device is operated by the controller. MOSFET is controlled with pulse width modulation technique with fixed switching frequency but with variation in pulse width.

\section{3) Controller Block}

Current mode control is well suited for power quality improvement, which actually senses and limits the peak inductor current and also allows input current to the desired sinusoidal wave shape. The current mode control can be implemented by different methods, namely: Peak current mode control (PCMC), PCMC with slope compensation, Peak to average current error control and Average current mode control (ACMC). For duty ratios exceeding 0.5 , peak current mode control is not suitable due to its stability problem resulting in sub-harmonic oscillations. A slope equal to the inductor current downslope has to be figured out in order to overcome this problem. The peak to average current error control is not suitable for boost topologies. Since the boost inductor is in input side, this control method distorts the input current waveform. To reduce this, the selected boost inductor should be large enough to reduce the ripple in currents. To increase the pf using Boost Converter operating in CCM mode, Inductor average current mode control is used in this paper.

\section{Simulations And Results}

\section{A. Simulink Model of Bridge Rectifier}

In most of the nonlinear devices, an AC-DC conversion is required which is basically done by a rectification unit. Considering variable frequency drive (VFD) as an example, the initial part of the circuit will be the rectification unit through which the conversion takes place. In order to understand the problem statement better, we have simulated a single phase bridge rectifier circuit with and without the capacitor filter in MATLAB software. Initially, the rectifier circuit was simulated without a capacitive filter; which resulted in rippled output DC. At the same time, the waveform of the input current remained undistorted. In order to mitigate the ripple effect a capacitor was provided across the output terminal.

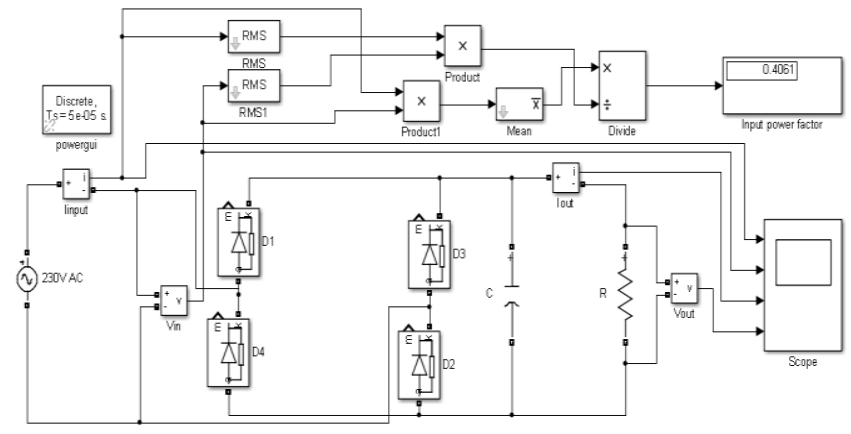

Fig.4. Simulink Model of Bridge Rectifier

This time we can observe that the input current waveform was affected by the injection of harmonics and the waveform was highly distorted. Since we had simulated a single phase rectifier, the 3rd harmonic component was dominating. As a result the power factor of the system was reduced due to the introduced distortion. The Simulink model of a single phase bridge rectifier is shown in Fig.4.

When the rectifier circuit was simulated without adding the capacitive filter, the input power factor was to be obtained 0.9214 . When the capacitive filter was added, the overall input power factor was reduced due to the harmonic distortion introduced in the input current. The power factor was reduced to a value of around 0.4061 when using a $200 \mu \mathrm{F}$ capacitor. As the value of the capacitive filter goes on increasing, the output becomes smoother whereas the input current becomes more and more distorted and accordingly reduces the power factor.

For a load of $100 \Omega$, the input power factor value varies for different values of capacitor provided.

Table.1. Variation in input power factor for different capacitor values.

\begin{tabular}{|c|c|}
\hline Capacitor Filter Value & Input Power Factor \\
\hline $10 \mu \mathrm{F}$ & 0.6714 \\
\hline $50 \mu \mathrm{F}$ & 0.5456 \\
\hline $100 \mu \mathrm{F}$ & 0.4294 \\
\hline $200 \mu \mathrm{F}$ & 0.4061 \\
\hline $500 \mu \mathrm{F}$ & 0.3229 \\
\hline
\end{tabular}

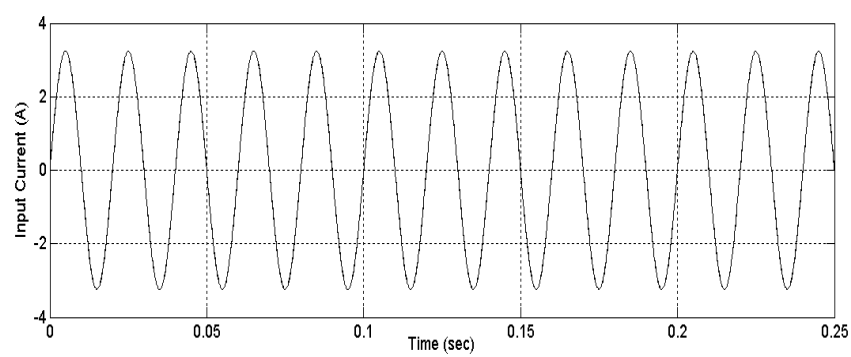

Fig.5. Input current waveform (without capacitive filter) 


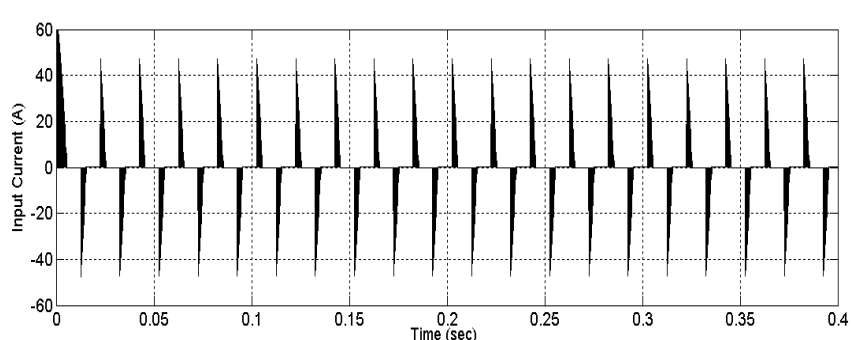

Fig.6. Input current waveform (with capacitive filter)

\section{1) FFT Analysis}

The FFT is a fast algorithm which can compute the discrete Fourier Transform of a sequence. Using FFT, we can observe the frequency content and the magnitude indicates the strength of frequency components relative to the fundamental components. When we perform FFT analysis on a waveform, we will be able to get information regarding the different order of harmonics which is present in that particular waveform. Also the Total Harmonic Distortion value can be obtained. The harmonic spectrum of the waveform of input current in the rectifier circuit with and without capacitor filter is shown below.
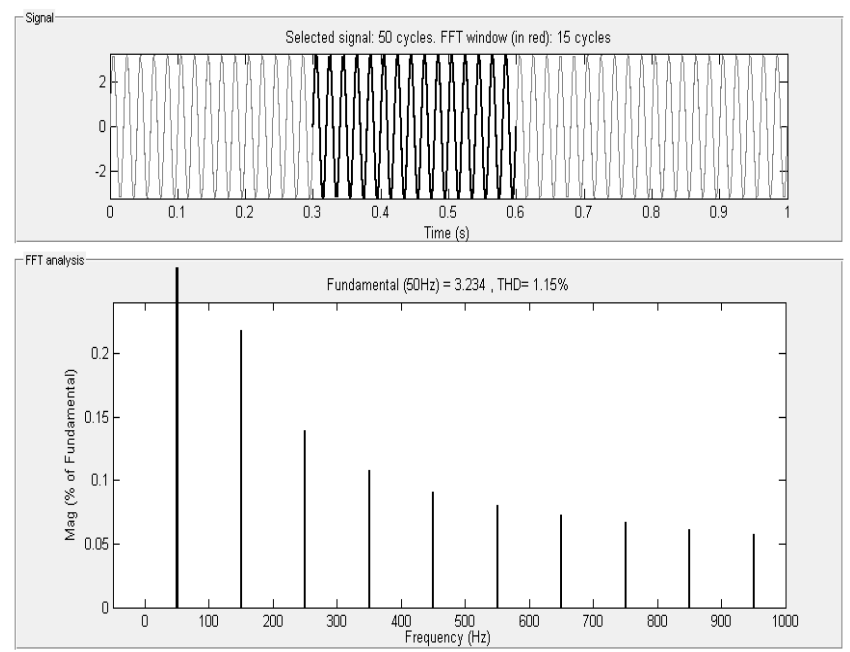

Fig.7. Harmonic Spectrum of input current (without capacitive filter)

In Fig.7, the fundamental component has a magnitude of 3.234 ; the third harmonic component is only around $0.22 \%$ of the fundamental component. Accordingly we get the THD= $1.15 \%$ which is within the tolerance value. As the order of harmonics goes on increasing, we can observe the magnitude of harmonics coming down.
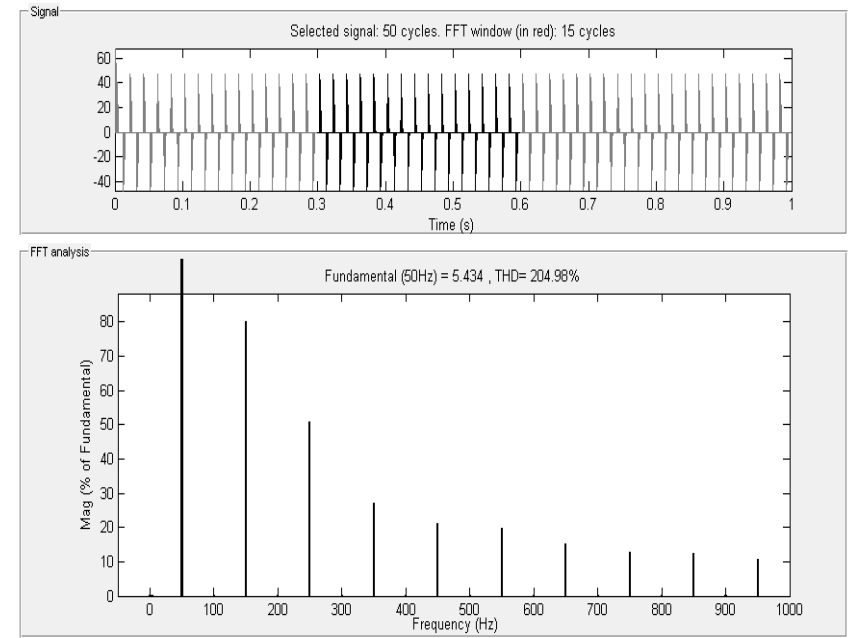

Fig.8. Harmonic Spectrum of input current (with capacitive filter)

The FFT graph shown in Fig.8 was obtained when using a $200 \mu \mathrm{F}$ capacitor filter. The percentage of harmonic components has increased due to non-linearity; $3^{\text {rd }}$ harmonics is almost $80 \%$ of the fundamental and there is a major increase in the THD which was obtained to be $204.96 \%$.

\section{B. Simulink Model of Active power filter using Boost Converter}

In this paper our focus is to mitigate the above power quality issue using a boost converter operated in CCM mode. The final proposed model of the active power filter system is a combination of rectifier and boost converter along with the gate driving circuit for the boost switch.

The final Simulink model of the system is shown in Fig. 11. The rectified output voltage from the bridge rectifier is given as the input to the boost converter circuit. In our system, the boost converter parameters are designed accordingly to provide a regulated output voltage of $400 \mathrm{~V}$. The boost converter circuit is being operated in the CCM mode, in which the inductor current will never drop to the zero line and is continuous while operating. The boost circuit is provided with the voltage control loop and the current control loop for providing the corresponding gate drive signals. The simulation of the circuit was done for $100 \mathrm{~W}$ load. The Simulink blocks provided at the bottom of the model are accountable for providing the gate driving signals for the switch provided in the boost circuit. The boost circuit is provided with the voltage control loop and the current control loop for providing the corresponding gate drive signals. The input current waveform is corrected by comparing the reference voltage waveform from the input voltage side and the inductor current waveform from the boost converter. The $\mathrm{S}-\mathrm{R}$ flip flop given in the model is provided with a switching frequency of $25 \mathrm{kHz}$ which accordingly controls the switching $\mathrm{ON}$ and OFF of the boost converter. By simulating this model, we were able to bring down the input current harmonics considerably. By reducing the harmonic effect and bringing the input current waveform back to almost pure sinusoidal, accordingly we were able to bring back the power factor close to unity. 


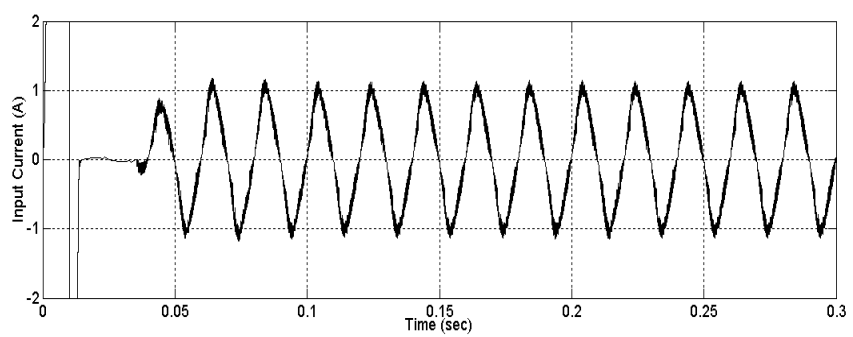

Fig.9. Improved Waveform of Input Current

The input current waveform which was improved by the active power filter is shown in Fig.9. We can observe that the current waveform has got back its sinusoidal waveform. So, accordingly we were able to observe that the power factor has been improved close to unity.

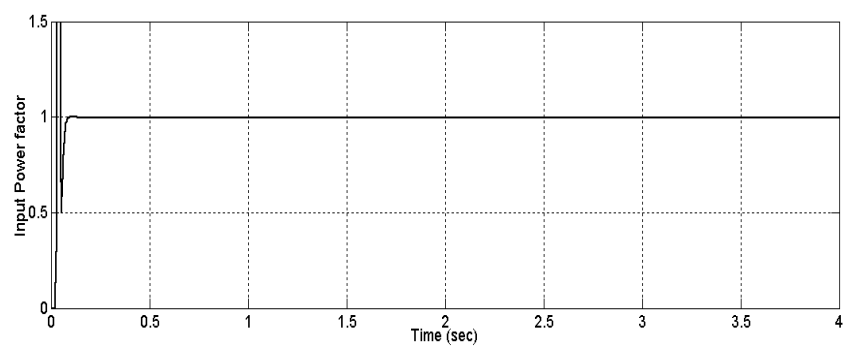

Fig.10. Improved input power factor 


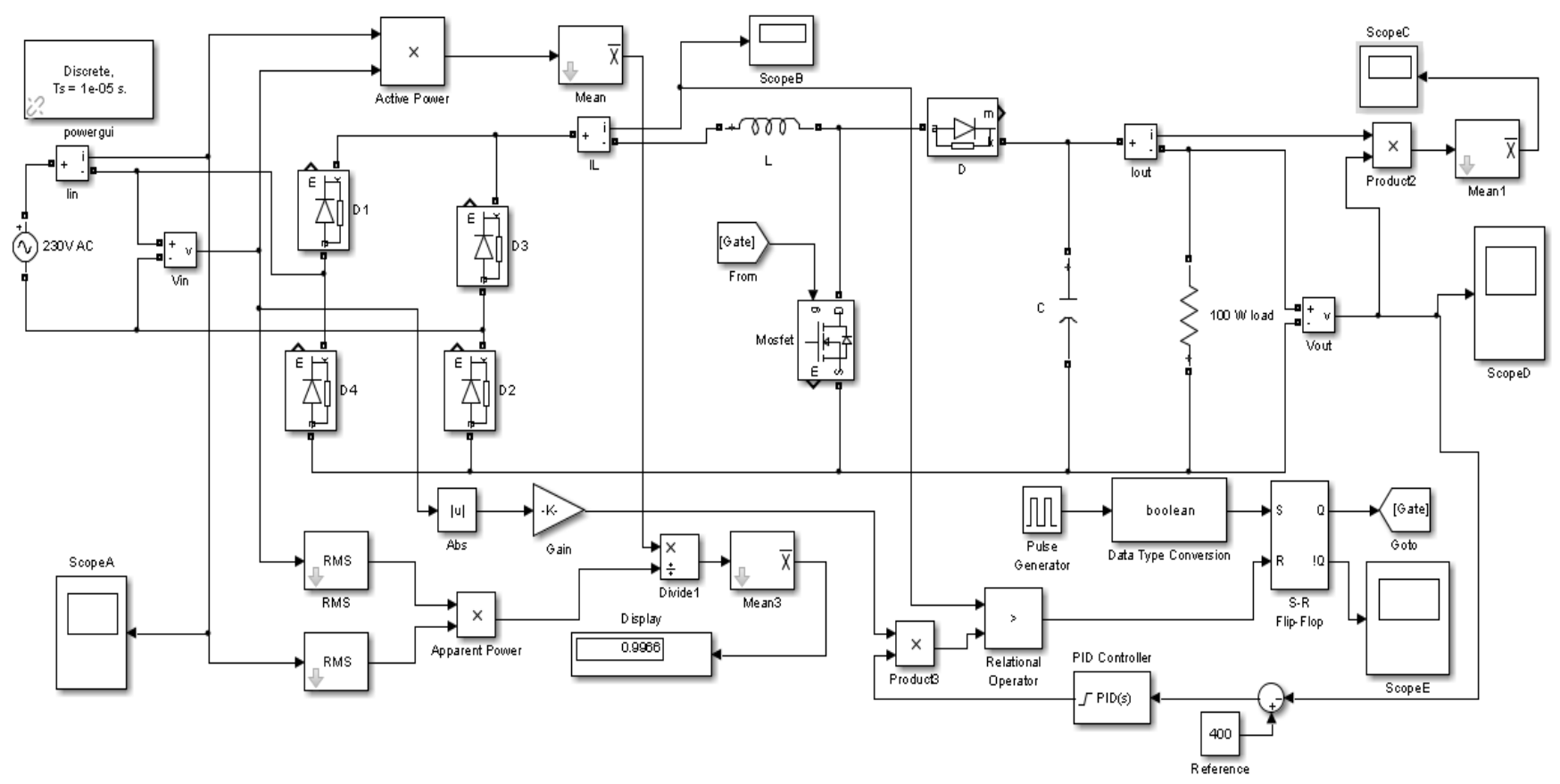

Fig.11. Simulink Model of Final Proposed Circuit

Fig.10 shows the improved input power factor. We can see that the input power factor has been improved to a value close to unity. This helps in reducing the harmonic losses and related effects.

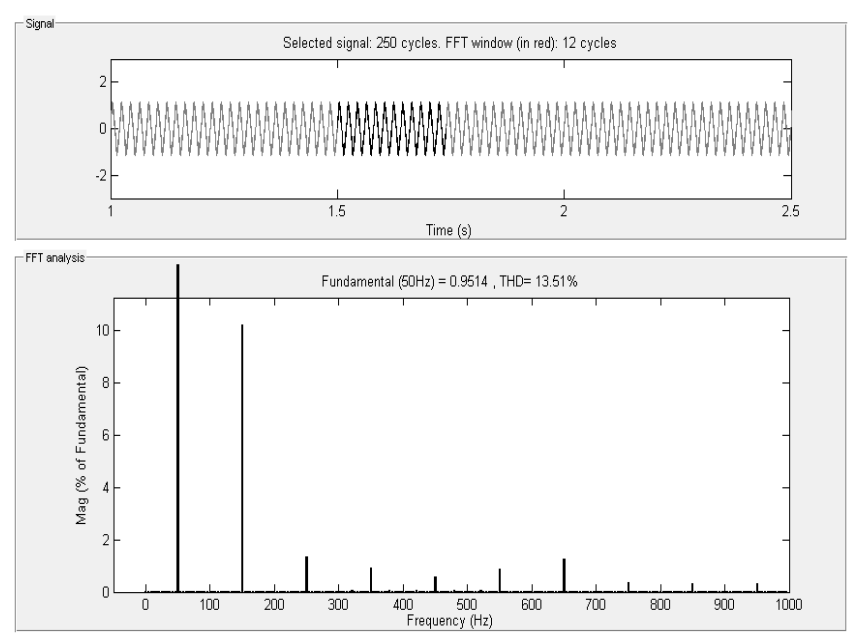

Fig.12. Harmonic Spectrum of Improved Current Waveform

Fig.12 shows the FFT analysis on the improved current waveform. By Observing the figure we can understand that the harmonic components magnitude has dropped drastically due to the filter used. The THD was obtained to be $13.51 \%$, clearly showing a decrease in total harmonic distortion.

\section{UCC28180 CONTROLLER}

The UCC28180 is a boost controller used for power factor correction operating at a fixed frequency in continuous conduction mode. It is flexible, easy-to-use and consists of 8-pin solution. It operates under Continuous Conduction Mode (CCM) to achieve high Power Factor. The UCC28180 can be operated as an active PFC pre-regulator with the help of external components. The maximum operating supply voltage is $21 \mathrm{~V}$ and supply current is $7 \mathrm{~mA}$. UCC28180 is ideal for universal $\mathrm{AC}$ input systems that operate in a range of $100 \mathrm{~W}$ to a few $\mathrm{kW}$ and switching frequency between $18 \mathrm{kHz}$ and $250 \mathrm{kHz}$. It supports both IGBT and power MOSFET switches. It employs two control loops. One is inner loop and other is outer loop. The inner current loop shapes the average input current to match the sinusoidal input voltage. The inner current loop avoids the need to sense input voltage by exploiting the relationship between input voltage and boost duty-cycle. An internal error amplifier and $5 \mathrm{~V}$ reference provide a slow outer loop to control the output voltage. External compensation of this outer loop is applied by means of the $\mathrm{V}_{\text {Comp }}$ pin. UCC28180 provides an enhanced dynamic response circuit that is based on the voltage feedback signal to improve response under fast load transients, both for output overvoltage and under voltage condition. It is ideally suited to minimize noise on the small signal traces. Other applications of this controller are Server and desktop power supplies, air conditioners, refrigerators, industrial power supplies (DIN Rail),flat panel TVs.

\section{PCB DESIGN}

A Printed Circuit Board mechanically supports and electrically connects the components using conductive tracks, etched from one or more sheet layers of copper laminated onto a non-conductive substrate. The PCB design software used here is Autodesk EAGLE.

The PCB schematic circuit shown in Fig.13 is the first part of designing a printed circuit board. The schematic consists of an upper part which involves the non-linear load and a lower part which shows the IC used for the power factor correction. The circuit begins with protective components a fuse, a thermistor and a varistor. The output from the rectifier is fed to the Boost converter. The converter consists of an inductor, a switching element and a diode. The output voltage is fed to pin number 6 of the PFC IC. A common ground is provided for the IC. The input value of the boost converter is fed to the IC pin number 3. The IC sets the frequency of switching as the pin number 4 is connected to the ground via a resistor. The pin number 8 which is associated with the gate drive 
sends the switching pulses to the MOSFET of the boost converter.

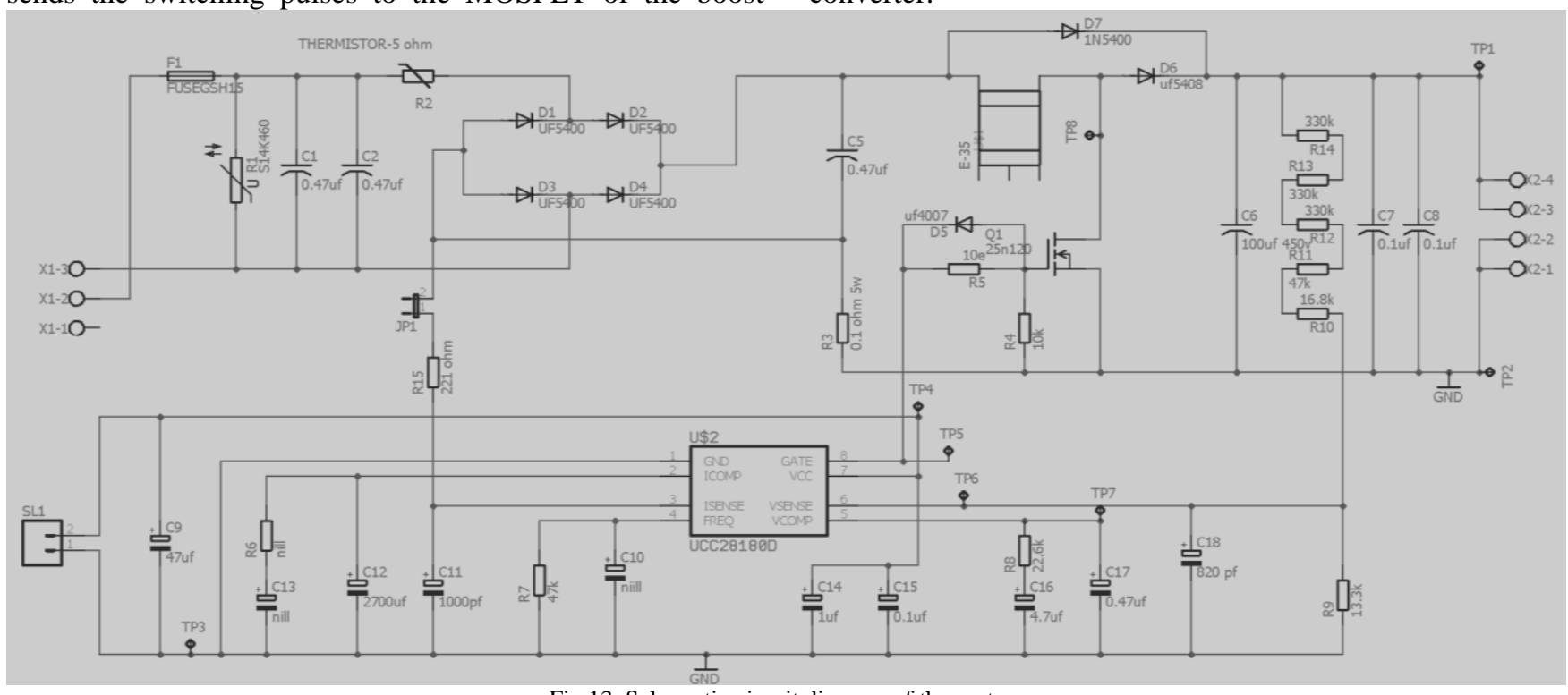

Fig.13. Schematic circuit diagram of the system

EAGLE's board designer is where the dimensions of the board come together; parts are arranged and connected by copper traces. By clicking the switch to board icon on the top toolbar the schematic was converted to PCB layout. All of the parts added from the schematic would be there, stacked on top of each other. The next step in PCB design is to align the components in a space effective manner. Improper component placement can lead to a horde of issues that affect functionality and durability of PCB. After placement, the routing step adds wires needed to properly connect the components while obeying all design rules for the IC. Once routing was completed the design was checked for error and finally deployed for printing.

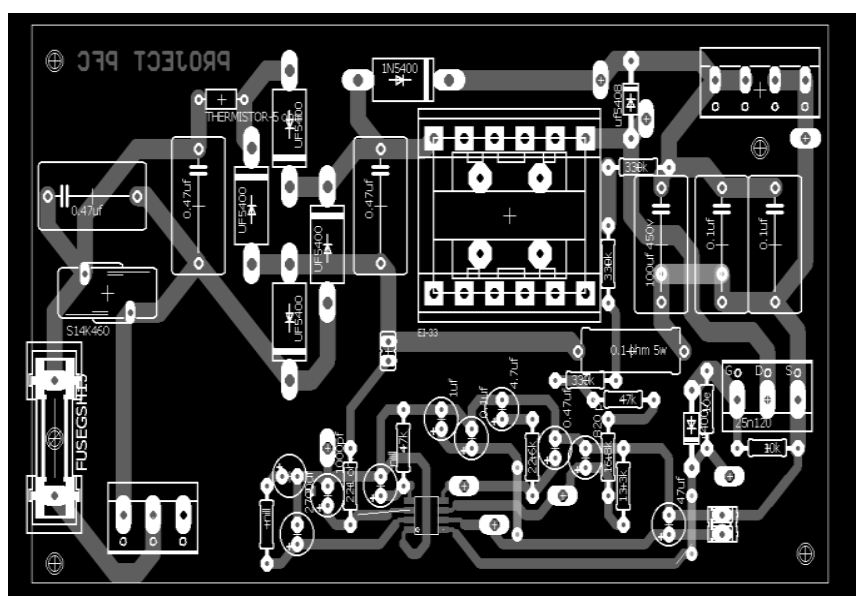

Fig.14. Board layout of schematic circuit

\section{CONCLUSION}

Waveform distortion is considered harmful as it can increase the effective peak value and also the RMS current in the connected load. The utility charges the industries with penalty for maintaining poor power factor. Thus, industries are keen to welcome technologies that reduce the energy losses as the pay heavily for the energy they use. The active power filter controller which could be an effective solution for the aforesaid issue was successfully simulated on
MATLAB, with the results clearly showing a considerable reduction in total harmonic distortion. An error free schematic and board layout was developed for the PCB design of the controller.

As a further advancement in the project, we can introduce bridgeless topology to the PFC circuit. The purpose behind such topology is to improve the power supply efficiency. The power factor controller circuit contain an input rectifier bridge. At any instant, the input current flows through three semiconductor devices- two diodes of the bridge rectifier and the boost switch. By means of a bridgeless topology the number of semi-conductor devices in the current path can be reduced and thereby the conduction power losses. It is also results in a simplified control circuit.

\section{REFERENCES}

[1] Periyaazhagar Devanathan and S. Irrusapparajan, "A survey of harmonic distortion and reduction techniques", International Journal of Pharmacy \& Technology (IJPT), vol. 8(4), pp.23581-23589, Dec 2016.

[2] H. Mageed, A. S. Nada, S. Abu-Zaid and R. S. Salah Eldeen,"Effects of waveforms distortion for household appliances on power quality", MAPAN-Journal of Metrology Society of India, Vol 34(4), pp.559-572, Nov 2019.

[3] Roohi Sachan and Rohan Srivastava,"Performance Analysis of fixed shunt passive filters harmonic mitigation", International Conference on Emerging Trends in Electrical, Electronics and Sustainable Energy Systems, 2016.

[4] Henning Tischer and Tomaz Pfeifer," Hybrid filter for dynamic harmonics filtering and reduction of commutation notches - A Case Study", $17^{\text {th }}$ International Conference on Harmonics and Quality of Power, Dec 2016.

[5] V. Suresh Kumar and V.Saravanan," A case study on harmonic distortion in textile industry", Australian Universities Power Engineering Conference (AUPEC), Nov 2015.

[6] A. T. Baitade and S. S. Chopade,"Harmonic reduction, power factor improvement and speed detection for 3-phase induction motor drive system", Conference on Advances in Signal Processing (CASP), June 2016.

[7] Mahesh Swamy and Anupama Balakrishnan," Three, Single-Phase Power Factor Correction (PFC) Boost Converters for Use with Three-Phase, 3-Wire Variable Frequency Drive Systems", IEEE Energy Conversion Congress and Exposition, Oct 2015.

[8] C. K. Neha, Manasa Hegde, Vikrant Aher, Vasudha Hegde,"Effect of home appliances on power quality of conventional grid", International Conference on Circuits, Controls, Communications and Computing, Oct 2016. 
[9] N. Rugthaicharoencheep and S. Chaladying,"Technical and financial evaluation for investment of harmonic mitigation in power network", IEEE Manchester PowerTech, June 2017.

[10] Mohamed Emad Farrag, Ayman Haggag, Haroon Farooq and Waqas Ali,"Analysis and mitigation of harmonics caused by air conditioners in a distribution system", $19^{\text {th }}$ International Middle East Power Systems Conference (MEPCON), Dec 2017.

[11] Wei Jiang, Yu-fei Zhou and Jun-ning Chen,'Modeling and Simulation of boost Converter in CCM and DCM", $2^{\text {nd }}$ International Conference on Power Electronics and Intelligent transportation System (PEITS), Dec 2009. 